\title{
Enhanced Adsorption of Cooking Fume Pollutants By Loofah Carbon Modified By Fenton Reagents
}

Huan Zhang

Guilin University of Technology

\section{Peng Zeng}

Guilin University of Technology

Hongquan Liu

Guilin University of Technology

Lei Liao ( $\square 976285986 @ q q . c o m$ )

Guilin University of Technology

Shengpeng Mo

Guilin University of Technology

Aimiao Qin

Guilin University of Technology

Jianwen Wei

Guilin University of Technology

Hongqiang Wang

Guilin University of Technology

\section{Research Article}

Keywords: Loofah carbon, Fenton's reagent, Adsorption, Oil, Particulate matter, Non-methane hydrocarbon

Posted Date: August 19th, 2021

DOl: https://doi.org/10.21203/rs.3.rs-629567/v1

License: (c) (1) This work is licensed under a Creative Commons Attribution 4.0 International License.

Read Full License 


\title{
Enhanced adsorption of cooking fume pollutants by loofah carbon modified by Fenton reagents
}

\author{
Huan Zhang ${ }^{1,3}$, Peng Zeng ${ }^{1,3}$, Hongquan Liu ${ }^{1,3}$, Lei Liao ${ }^{1,3, *}$, Shengpeng Mo ${ }^{1,3}$, Aimiao Qin², \\ Jianwen Wei ${ }^{1,3}$, Hongqiang Wang ${ }^{1,3}$ \\ 1. College of Environmental Science and Engineering, Guilin University of Technology, Guilin 541004, China; \\ 854488470@qq.com (H.Z.); 1046161013@qq.com (P.Z.); 122814686@qq.com (H.L.); moshengpeng14@mails.ucas.ac.cn \\ (S.M.); jianwen988@126.com (J.W.); 543393918@qq.com (H.W.) \\ 2. College of Materials Science and Engineering, Guilin University of Technology, Guilin 541004, China; \\ 2005032@glut.edu.cn (A.Q.) \\ 3. Guangxi Key Laboratory of Environmental Pollution Control Theory and Technology, Guilin University of Technology, Guilin \\ 541004, China. \\ * Correspondence: 976285986@qq.com (L.L.)
}

\begin{abstract}
In this study, natural loofah was used as a raw material to adsorb cooking fume pollutants after grinding into a powder (TGS), activation by phosphoric acid to generate activated loofah carbon (TGSC-0), and further modification by Fenton's reagent (TGSC-1). SEM, GC-MS, FT-IR, and X-ray diffraction analyses, in addition to surface area and pore measurements, were used to characterize the adsorption performance of TGS, TGSC-0, and TGSC-1 toward cooking fume pollutants including oils, particulate matter, and non-methane hydrocarbon). TGSC-1 was the best adsorbent when compared against TGS and TGSC-0, and exhibited saturated adsorption capacities for oil, non-methane hydrocarbon (NMHC), PM2.5, and PM10 of $10.367 \mathrm{mg} / \mathrm{g}, 4.132 \mathrm{mg} / \mathrm{g}, 5.613 \mu \mathrm{g} / \mathrm{g}$, and $16.486 \mu \mathrm{g} / \mathrm{g}$, respectively. Microscopy indicated that the TGSC-1 surface was rougher than that of TGSC-0. In addition, the adsorption properties of TGSC-1 were enhanced due to abundant hydroxyl, carbonyl, and carboxyl groups on the material surfaces, while iron was also present in the amorphous form that was generated on TGSC-1 surfaces from Fenton's reagent. As TGSC-1 mass increased, the adsorption breakthrough time and adsorption capacity for simulated cooking fumes (SCFs) gradually increased. Further, Langmuir models better fit the adsorption process based on the highest $\mathrm{R}^{2}$ values being observed for Langmuir model fitting curves of TGSC-1 adsorption of pollutants (i.e., oils, NMHC, PM2.5, and PM10) from SCF, suggesting that adsorption was primarily due to monolayer adsorption and that chemical adsorption plays a major role in this process. This study provides a theoretical basis for the application of TGSC adsorption technology in the treatment of cooking fumes.
\end{abstract}

Keywords: Loofah carbon; Fenton's reagent; Adsorption; Oil; Particulate matter; Non-methane hydrocarbon. 


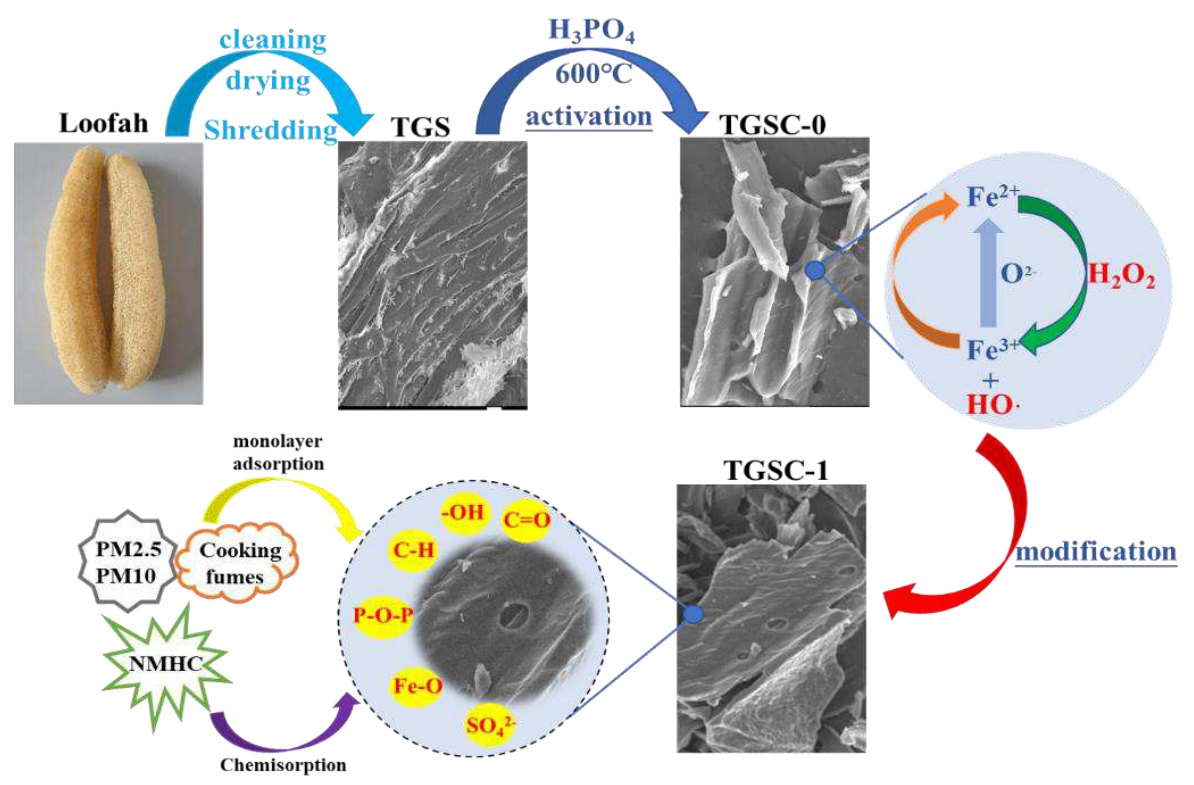

\section{Introduction}

Increased economic development and improved living standards have led to a focus on preventing and controlling resultant air pollution for environmental protection (Ma et all. 2020). The Chinese catering industry has rapidly developed in recent years and the associated cooking fumes have become the primary source of indoor air pollution. Cooking fumes represent a unique source of pollution in China and have gradually become the third largest polluting gas in recent years, behind industrial waste gas and automobile exhaust (Alves et all. 2021).

Cooking fumes contain carcinogens, although carcinogens like formaldehyde, acetaldehyde, and benzene are not detected in edible oils and fuels, suggesting that these compounds entirely derive from cooking activities ( $\mathrm{Yu}$ et all. 2015). High-temperature cooking has become more popular in recent years to disinfect and improve food tastes, but this style of cooking also produces more cooking fumes (Cheng et all. 2016). Cooking fume contains a complex mixture of toxic pollutants including metals, benzene, particulate matter (PM), volatile organic compounds, polycyclic aromatic hydrocarbons (PAHs), quinones, and carbonyl compounds. Consequently, exposure to oil fumes can lead to various health effects including heart and lung diseases (Abdullahi et all. 2013; Wong et all. 2013), diabetes (Wang et all. 2018), brain damage (Naseri et all. 2019), sleep disorders (Fu et all. 2017), female reproductive problems (Zhang et all. 2020), and even diseases in children resulting from contact with mothers during pregnancy (Fang et all. 2020). Cooking fume waste gas is a multiphase aerosol fluid suspended in air that combines gas, liquids, and solids. Waste gas density usually ranges from 1,130 to $11,134 \mathrm{~kg} / \mathrm{m}^{3}$ and exhibits characteristic expansibility, fluidity, boundary layer effects, and viscosity (Zhao et all. 2019). Thus, cooking fumes contain a variety of toxic and harmful components that vary with cooking methods, food types, and heating temperatures. In general, cooking fumes comprise mixed gases that form from thermal volatilization, oxidation, pyrolysis, and polymerization of edible oils and ingredients at high temperatures, in addition to their secondary reactants (Zhang et all. 2019). Therefore, cooking fumes represent compound pollutants with complex and variable compositions, and their composition varies with changes in temperature. The current primary monitoring focus of cooking fume pollutants is the examination of gas phase concentrations of cooking fumes and benzene series (Tucki et all. 2019). For example, experimental collection of cooking fume samples from different restaurants have shown that 
waste gas from barbecue restaurants primarily comprise alkane olefins, while home-cooked dishes primarily produce isobutane or propylene, and fried dishes primarily produce olefins (Ho et all. 2019). When high-temperature cooking fumes are emitted into the atmosphere, some VOCs can be converted into new particles through homogeneous or heterogeneous reactions (Li et all. 2011; Wang et all. 2015). Therefore, evaluation of cooking fume pollutants should not be limited to gas phase concentrations of cooking fumes, but should also include concentrations of NMHC in addition to particulate matter (PM2.5 and PM10) within catering cooking fumes.

Activated carbon is the most common adsorbent and is widely used in various applications due to its porous structure and active groups on its surface (Ho et all. 2021). The adsorption performance of activated carbon is determined by its surface structure and chemical properties. Modification of carbon surfaces by activation can improve their adsorption performance. However, the popularization and application of activated carbon is limited due to its high costs. Thus, studies have focused on identifying low-cost and easily available agricultural waste materials to prepare activated wooden carbon with excellent adsorption effects (Wang et all. 2021; Ali et all. 2021). Activated carbon can be produced from rice straw, fruit shells, and bagasse raw materials, while applied activators are generally $\mathrm{KOH}$, $\mathrm{ZnCl}_{2}$, and $\mathrm{H}_{3} \mathrm{PO}_{4}$ (Liu et all. 2021). In addition, phosphoric acid has been widely used as an activator due to its high carbon yield and low energy consumption (Ali. et all. 2020).

Few studies have investigated the use of loofah to prepare activated carbon and no studies have evaluated the modification of materials with Fenton reagents. In this study, natural loofah was investigated as a raw material with phosphoric acid as an activator to prepare loofah carbon. Fenton reagent modification successfully led to the generation of a new type of loofah carbon material that was subsequently characterized using SEM, FT-IR, XRD, and $\mathrm{N}_{2}$ adsorption-desorption modeling. These new results provide a scientific basis for developing agricultural waste to mitigate cooking fume pollutants.

\section{Materials and methods}

\subsection{Materials}

Natural loofah was purchased from Jinan within Shandong, China. Perchloroethylene (purity $\geq$ 99.0\%), 30\% hydrogen peroxide, phosphoric acid (purity $\geq 99.0 \%$ ), iron(II) sulfate heptahydrate (purity $\geq 99.0 \%$ ), and anhydrous sodium sulfate (purity $\geq 99.0 \%$ ) were all purchased from the Shanghai Aladdin Biochemical Technology Co., Ltd., China company. Vegetable blend, chili, and zanthoxylum oils were provided by Golden Dragon Fish Food Co., Ltd., China. Condensate waste oil was retrieved from a canteen at the Guilin University of Technology, China.

Sample morphologies were investigated with a JSM-6380LV scanning electron microscope (JEOL, Japan). The skeletons and functional groups within sample compounds were characterized with a ThermoNexus 470 Fourier infrared spectrometer (Nicolet, United States). XRD analysis was conducted using an $X^{\prime}$ PertPRO X-ray powder diffraction instrument (PANalytical, Netherlands). Nitrogen adsorption and desorption isotherms were measured with a ASAP2020 automatic rapid specific surface area and mesopore / micropore analyzer (Micromeritics, United States). The concentrations of pollutants were determined with a JC-OIL-6A infrared oil meter (Qingdao Juchuang Environmental Protection Co., Ltd., China), a portable SKY2000 non-methane total hydrocarbon meter (Shenzhen Yuante Technology Co., Ltd., China), and a CW-HAT portable particulate matter meter (Background Yashiruifeng Purification Technology Co., Ltd., China).

\subsection{Preparation of activated loofah carbon}


Natural loofah was washed with distilled water and then dried in an oven at $105^{\circ} \mathrm{C}$ for $12 \mathrm{~h}$, followed by shearing into fragments and grinding into a powder. The powder was then cleaned with distilled water until reaching a neutral $\mathrm{pH}$, vacuum filtered, and then oven dried at $105^{\circ} \mathrm{C}$ for $6 \mathrm{~h}$. The dried powder was sieved through a 100 mesh sieve and particulate matter $(\varphi=150 \mu \mathrm{m}$; i.e., the original loofah powder) was identified as the towel-gourd sponge power (TGS). The TGS (20 g) was mixed with a $60 \%$ phosphoric acid solution at a material to liquid ratio of 1:5 (w: w) for $24 \mathrm{~h}$. The powder was then vacuum filtered and dried in an oven at $105^{\circ} \mathrm{C}$ for $6 \mathrm{~h}$. The dried loofah powder mixed with phosphoric acid was placed in a muffle furnace and isolated from oxygen carbonization at $600^{\circ} \mathrm{C}$ for $2 \mathrm{~h}$, followed by washing with distilled water until reaching a neutral $\mathrm{pH}$, drying, and filtering through a 100-mesh sieve. The resulting loofah charcoal material was identified as TGSC-0. Twenty milliliters of $\mathrm{FeSO}_{4}(0.5 \mathrm{~mol} / \mathrm{L})$ were added to the beaker containing the material, and $\mathrm{H}_{2} \mathrm{O}_{2}$ was added at an $\mathrm{H}_{2} \mathrm{O}_{2}$ to $\mathrm{Fe}^{2+}$ ratio of 10:1. The $\mathrm{pH}$ was then adjusted to 3-4 by adding $10 \mathrm{~mL}$ of $\mathrm{H}_{2} \mathrm{O}_{2}$ to configure the Fenton's reagent. The mixture of $5 \mathrm{~g}$ TGSC-0 and Fenton's reagent was stand for $24 \mathrm{~h}$, cleaned with distilled water until the $\mathrm{pH}$ was neutral, and vacuum filtered. Then, the powder was placed in a drying oven for $6 \mathrm{~h}$ at $105^{\circ} \mathrm{C}$. The resulting Fenton-modified loofah carbon material was identified as TGSC-1.

\subsection{Adsorption of pollutants from cooking fumes}

A schematic of the adsorption device is shown in Fig. 1. The self-constructed cooking fume waste gas was used to simulate real-world fume gas. Specifically, $500 \mathrm{~mL}$ of blending oil, $20 \mathrm{~mL}$ of chili oil, $20 \mathrm{~mL}$ of zanthoxylum oil, and $60 \mathrm{~mL}$ of condensate waste oil were mixed well in a $1 \mathrm{~L}$ three-necked flask. The oil temperature was controlled by an electric stove and heated to $190^{\circ} \mathrm{C}$. The air pump passed air into the three-necked flask at a specific pressure and the air was mixed with the cooking fumes produced by heating to form simulated cooking fumes (SCF). A rotameter was used to control the inlet flow rate of flue $\left(\mathrm{Q}=0.12 \mathrm{~m}^{3} / \mathrm{h}\right)$ in addition to measuring the initial concentration of each pollutant in the cooking fumes at the inlet sampling port. The concentrations of oil, PM2.5, PM10, and NHMC were 100 $\mathrm{mg} / \mathrm{m}^{3}, 110 \mu \mathrm{g} / \mathrm{m}^{3}, 240 \mu \mathrm{g} / \mathrm{m}^{3}$, and $50 \mathrm{mg} / \mathrm{m}^{3}$, respectively. TGS, TGSC-0, and TGSC-1 (5 g) were individually placed in three double-sphere adsorption tubes (DSAT). The cooking fumes were fed to the DSAT, and the SCF pollutants were adsorbed with TGS, TGSC-0, and TGSC-1. In addition, the outlet concentrations of particulate matter (PM2.5/PM10) and NHMC were measured with portable PM2.5/PM10 and NHMC instruments, respectively. The outlet SCF samples were suctioned into a 25 $\mathrm{mL}$ syringe and then injected into $25 \mathrm{~mL}$ colorimetric tubes filled with $15 \mathrm{~mL}$ of perchloroethylene, filtered with qualitative filter paper, and dehydrated with anhydrous sodium sulfate, followed by measurements of oils with an infrared oil meter.

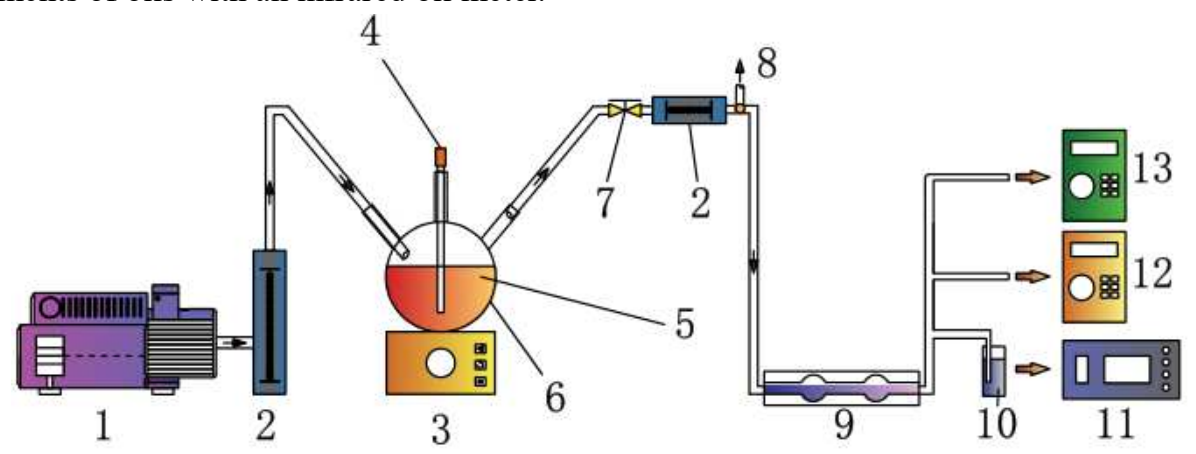

Fig. 1. Experimental schematic.

1: air pump, 2: rotameter, 3: electric stove, 4: thermocouple, 5: miscella, 6: three-necked flask, 7: 


\subsection{Experimental methods}

\subsubsection{Adsorption curve and adsorption capacity calculations}

The changes in outlet concentrations of oil, particulate matter (PM10/PM2.5), and NMHC from SCF were used to evaluate adsorption times. Specifically, adsorption times were used as the abscissa and outlet oil concentrations from SCF were used as the ordinates to calculate TGS, TGSC-0, and TGSC-1 adsorption curves for pollutants. A $2.0 \mathrm{mg} / \mathrm{m}^{3}$ maximum allowable emission concentration was set as the breakthrough point of oil concentration according to Chinese catering industry standards. The time corresponding to the outlet concentration/inlet concentration ratio of 0.05 was the breakthrough time for the concentration of particulate matter and NMHC and can be used to calculate the breakthrough adsorption capacity. The time corresponding to the outlet concentration/inlet concentration ratio 0.95 was set as the saturated adsorption time for each pollutant, and the respective saturated adsorption capacity was also calculated.

The adsorption capacity for pollutants from SCF was determined using Eq. (E1).

$$
\mathrm{q}=\frac{\left(\mathrm{C}_{0} \cdot \mathrm{t}-\mathrm{S}\right) \cdot \mathrm{Q}}{\mathrm{M}},
$$

where $\mathrm{q}(\mathrm{mg} / \mathrm{g})$ is the adsorption capacity for the unit mass of adsorbent at $\mathrm{t}, \mathrm{C}_{0}(\mathrm{mg} / \mathrm{L})$ is the inlet concentration of pollutants, $\mathrm{S}$ is the integral area between the breakthrough adsorption curve and the abscissa, $\mathrm{t}(\mathrm{min})$ is the adsorption time, and $\mathrm{M}(\mathrm{g})$ is the mass of the adsorbent (Zietzschmann. et all. 2016).

\subsubsection{Wurof equation calculations}

The Wurof equation was used to explain changes of adsorption time and adsorption capacity for adsorbents with different masses to compound pollutants. The equation is as shown in Eq. (E2):

$$
\tau=\mathrm{K} \cdot \mathrm{L}-\tau_{0}
$$

where $\tau(\mathrm{min})$ is the adsorption breakthrough time, $\mathrm{K}(\mathrm{min} / \mathrm{m})$ is the adsorption layer protection coefficient, $\mathrm{L}(\mathrm{m})$ is the adsorption layer thickness, and $\tau_{0}(\mathrm{~min})$ is the protection time loss. $\mathrm{L}$ is calculated according to Eq. (E3)

$$
\mathrm{L}=\frac{\mathrm{m}}{\mathrm{A} \cdot \rho_{\mathrm{b}}},
$$

where $\mathrm{m}(\mathrm{kg})$ is the mass of adsorbent, $\mathrm{A}\left(\mathrm{m}^{2}\right)$ is the cross-sectional area of the adsorption layer, and $\rho_{\mathrm{b}}$ $\left(\mathrm{kg} / \mathrm{m}^{3}\right)$ is the bulk density of the adsorbent. The bulk density can be calculated by Eq. (E4):

$$
\rho_{\mathrm{b}}=(1-\varepsilon) \cdot \rho_{\mathrm{p}},
$$

where $\varepsilon$ is the voidage of the adsorption layer and is generally $0.35-0.7$, and $\rho_{p}\left(\mathrm{~kg} / \mathrm{m}^{3}\right)$ is the density of the adsorbent.

\subsubsection{Adsorption Isotherm Models}

Adsorption isotherms were described by Langmuir and Freundlich models that reflect the effects of concentration and temperature on adsorption properties. Langmuir models are often used to describe adsorption forms of monolayers and are suitable for adsorbents with uniform surfaces without

$$
\mathrm{q}_{\mathrm{e}}=\frac{\mathrm{q}_{\mathrm{m}} \cdot \mathrm{K}_{\mathrm{L}} \cdot \mathrm{C}_{\mathrm{e}}}{1+\mathrm{K}_{\mathrm{L}} \cdot \mathrm{C}_{\mathrm{e}}}
$$


where $\mathrm{q}_{\mathrm{e}}(\mathrm{mg} / \mathrm{g})$ is the adsorption capacity, $\mathrm{q}_{\mathrm{m}}(\mathrm{mg} / \mathrm{g})$ is the maximum adsorption capacity, $\mathrm{C}_{\mathrm{e}}(\mathrm{ppm})$ is the concentration of gas at saturation, and $\mathrm{K}_{\mathrm{L}}\left(\mathrm{ppm}^{-1}\right)$ is the Langmuir constant (Zhao. et all. 2020).

The Freundlich equation can be used to describe the adsorption isotherm of a heterogeneous surface adsorbent and is an empirical adsorption isotherm that is calculated as follows:

$$
\mathrm{q}_{\mathrm{e}}=\mathrm{K}_{\mathrm{F}} \cdot \mathrm{C}_{\mathrm{e}}{ }^{1 / \mathrm{n}} \text {, }
$$

where $K_{F}\left(\mathrm{mg} \cdot \mathrm{g}^{-1} \mathrm{ppm}^{-1 / \mathrm{n}}\right)$ is the Freundlich constant and $1 / \mathrm{n}$ is the heterogeneity factor (Novi Sri. et all. 2021).

\section{Results and discussion}

\subsection{Characterization of loofah adsorbents}

\subsubsection{SEM analysis}

SEM was used to characterize the surface microstructures of the TGS, TGSC-0, and TGSC-1 adsorbents (Fig. 2). The outer surfaces of TGSC-0, TGSC-1, and TGS exhibited corrugated structures previously described as "vascular bundle structures" (Liang. et all. 2021). In contrast, the TGS exhibited a rough surface with a large amount of attached pectin layers and an even distribution of Si (Zhang. et all. 2019). The TGSC-0 surface was flat and smooth, exhibiting abundant uniformly-distributed pore structures due to high temperature dehydration (Chen. et all. 2016) that played a role in the adsorption of pollutants from SCF. The TGSC-1 surface was rougher than that of TGSC-0 and exhibited abundant pores in addition to coverage by compounds formed by the Fenton reagent near the pores.

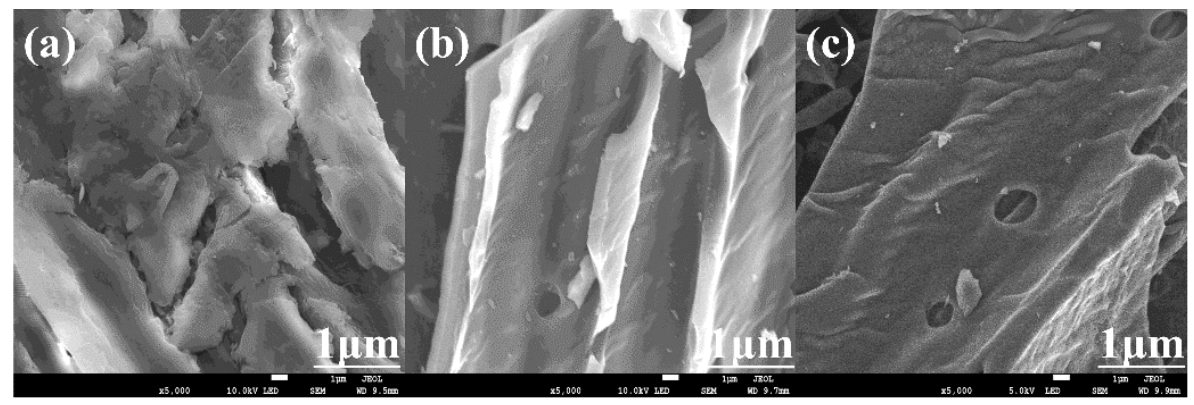

Fig. 2. SEM images of (a) TGS, (b) TGSC-0, and (c) TGSC-1 surfaces.

\subsubsection{Fourier infrared spectroscopy}

Infrared spectroscopy was conducted to further characterize the TGS, TGSC-0, and TGSC-1 materials (Fig. 3). The FT-IR spectra could be divided into two types of bands: one being the absorption band formed by pore-adsorbed water and - $\mathrm{OH}$ groups on the surface, and the other from skeleton vibrations (Cui. et all. 2021). The strong and wide absorption peaks near 3,436 cm-1 and 3,202 $\mathrm{cm}^{-1}$ (Fig. 3 ) are related to the stretching vibrations of $-\mathrm{OH}$. The absorption peak near $1,615 \mathrm{~cm}^{-1}$ represents the bending vibration peak of the physically-adsorbed water hydroxyl that was caused by the introduction of water molecules into the adsorbed water and interlayer pores on particle surfaces (Song. et all. 2020). The absorption peak near $2,923 \mathrm{~cm}^{-1}$ primarily derives from the asymmetric stretching vibrations of methylene $\mathrm{C}-\mathrm{H}$ bonds of alkyl groups. A peak near $1,714 \mathrm{~cm}^{-1}$ was also observed that corresponds to the stretching vibration peak of $\mathrm{C}=\mathrm{O}$ from carboxyl groups (Dai. et all. 2018). The prominent peak near $1,384 \mathrm{~cm}^{-1}$ corresponded to the stretching vibration peak of -NO. The spectral peaks of TGSC-0 and TGSC-1 after activation by phosphoric acid at 1,300-1,100 $\mathrm{cm}^{-1}$ were caused by phosphorus-containing functional groups, while the spectral peaks at $875 \mathrm{~cm}^{-1}$ corresponded to symmetrical vibrations of P-O$\mathrm{P}$ groups in polyphosphates (Shen. et all. 2015). In addition, spectral peaks were observed near 1,036 $\mathrm{cm}^{-1}$ that correspond to $\mathrm{SO}_{4}{ }^{2-}$. A spectral peak near $592 \mathrm{~cm}^{-1}$ was observed that corresponds to the lattice vibrations of cations $\left(\mathrm{Fe}^{3+}, \mathrm{Al}^{3+}\right.$, and $\left.\mathrm{Mg}^{2+}\right)$, consistent with the position of a characteristic stretching 
vibration peak for Fe-O (Ramalakshmi. et all. 2021). Thus, these results indicate that the loofah charcoal modified by Fenton reagent comprises a large number of hydroxyl, carbonyl, and carboxyl groups that can effectively improve the adsorption effects of loofah charcoal.

Fenton reactions use $\mathrm{Fe}^{2+}$ as a catalyst to react with $\mathrm{H}_{2} \mathrm{O}_{2}$ at $\mathrm{pH}=2-4$ in order to form $\mathrm{HO}$. groups with strong oxidation ability. Active groups can be introduced after surface modification of TGSC-0 via HO- formation mechanisms as follows (Vasilyeva. et all. 2021; Guo. et all. 2021):

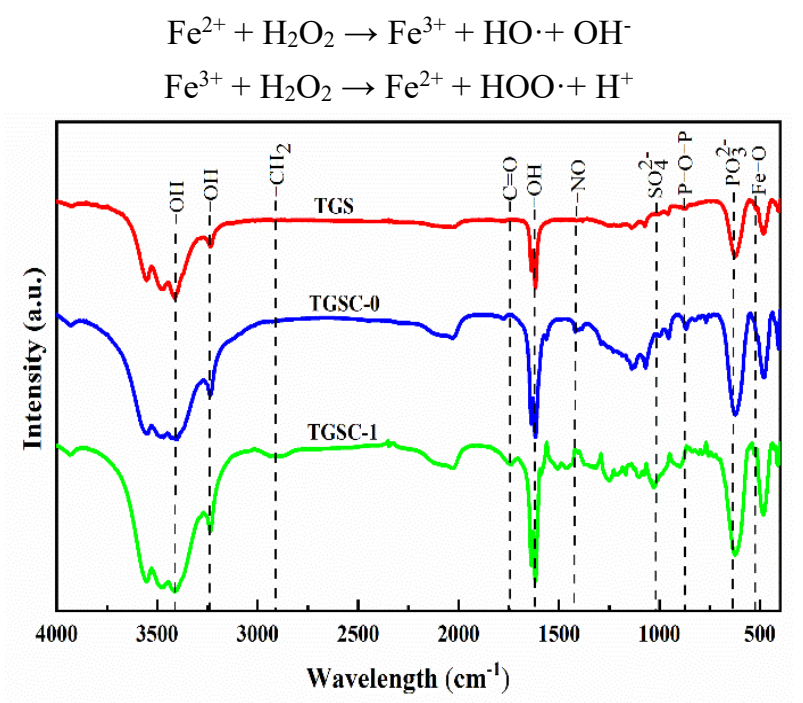

Fig. 3. FT-IR spectra of TGS, TGSC-0, and TGSC-1 materials.

\subsubsection{X-ray diffraction analysis}

XRD analysis was used to investigate the chemical composition of the TGS, TGSC-0, and TGSC1 materials (Fig. 4). A clear diffraction peak within the TGS spectra corresponded to a carbon diffraction peak at $15-25^{\circ}$ that could be attributed to an abundance of cellulose, lignin, and pectin on TGS surfaces (Fu. et all. 2018). These components are subject to modification under pyrolysis at high temperatures, leading to decreased diffraction peaks for TGSC-0 and TGSC-1. Comparison to JCPDS standards (No.06-0696) indicated that the peaks at $44.6^{\circ}$ and $64.8^{\circ}$ belonged to diffraction peaks from bodycentered cubic $\alpha$-Fe. The lack of iron-bearing mineral crystals in the corresponding spectra indicated that the loaded iron exists in an indefinite form and derives from the Fenton reagent reactions during TGSC0 modification.

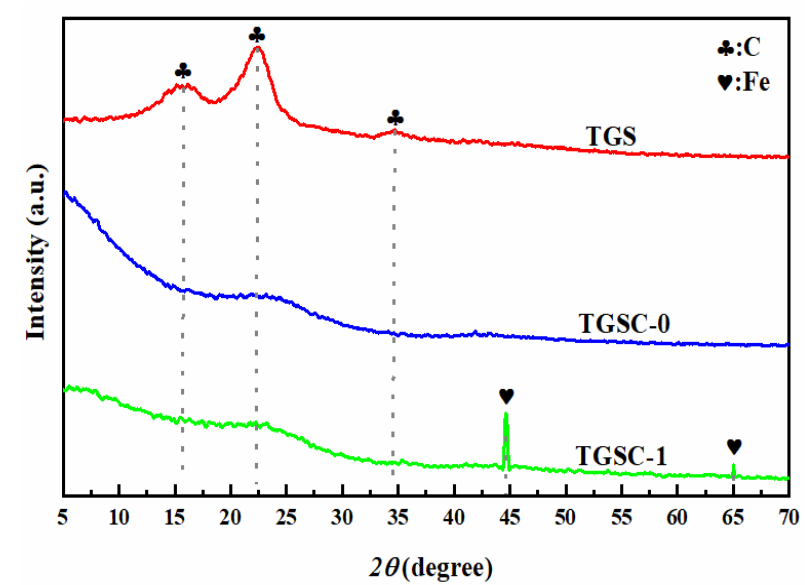

Fig. 4. XRD patterns of TGS, TGSC-0, and TGSC-1 materials. 


\subsubsection{BET analysis}

$\mathrm{N}_{2}$ adsorption-desorption isotherms were evaluated for TGS, TGSC-0, and TGSC-1 (Fig. 5). Based on IUPAC definitions, all three loofah adsorbents exhibited obvious type II adsorption isotherms and clear hysteresis loops. TGS exhibited a H3 hysteresis loop, while TGSC-0 and TGSC-1 exhibited H4 hysteresis loops. The adsorption-desorption curves were not consistent and exhibit obvious hysteresis loops, indicating that mesopores and macropores were present in the adsorption materials (Bardestani. et all. 2019). The curves of the two loofah carbon types indicated that adsorption capacity increased with increasing relative pressure, while adsorption capacity sharply increased at a relative pressure close to 1.0. The primary reason for this observation was that micropores of adsorbents were filled with $\mathrm{N}_{2}$ at low relative pressure, while adsorption gradually shifted from single-layer to multi-layer dynamics with increasing pressure. Finally, the adsorbent macropores were filled with $\mathrm{N}_{2}$, and a capillary agglomeration occurred at high relative pressure (Sinha. et all. 2019). The adsorption capacity of the three adsorbents followed the trend of TGSC-0 > TGSC-1 > TGS (Fig. 5).

Pore size distributions were evaluated for TGS, TGSC-0, and TGSC-1 (Fig. 6) and were uniform for TGSC-0 and TGSC-1 and primarily distributed in the range of $0.5-3.5 \mathrm{~nm}$, while that of TGS was primarily in the range of $1.6-4.0 \mathrm{~nm}$. The pore sizes of TGSC-1 were smaller than those of TGSC-0, primarily due to hydroxyl groups and iron ions that were grafted to the inner surface of the pores after Fenton reagent modification.

The specific surface area and pore structure parameters of TGS, TGSC-0, and TGSC-1 are shown in Table 1. The BET specific surface areas of the three adsorbents followed the trend of: TGSC-0 > TGSC-1 > TGS. After loofah carbon (TGSC-0) surface modification by Fenton's reagent, the pore structures were destroyed and the specific surface areas and pore volumes decreased (Zheng. et all. 2021). The average pore sizes of TGSC-0 and TGSC- 1 were $3.963 \mathrm{~nm}$ and $2.347 \mathrm{~nm}$, respectively.

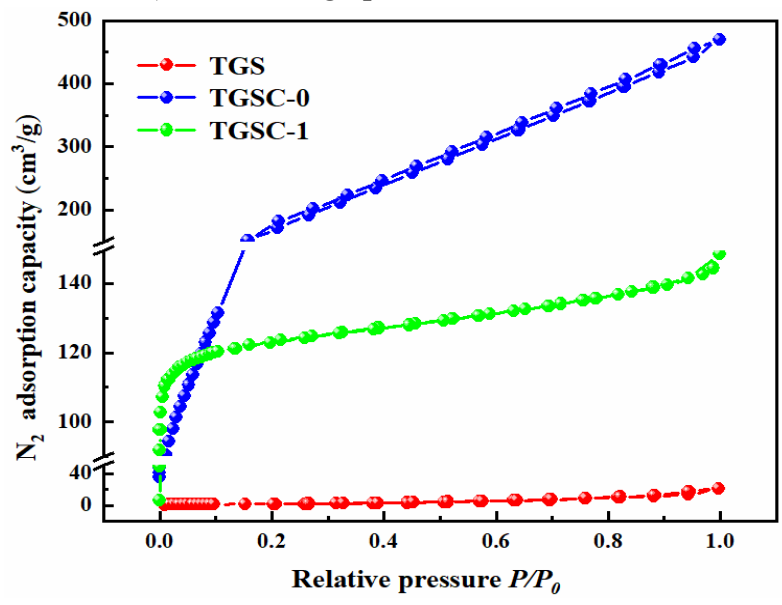

Fig. 5. $\mathrm{N}_{2}$ adsorption-desorption isotherm

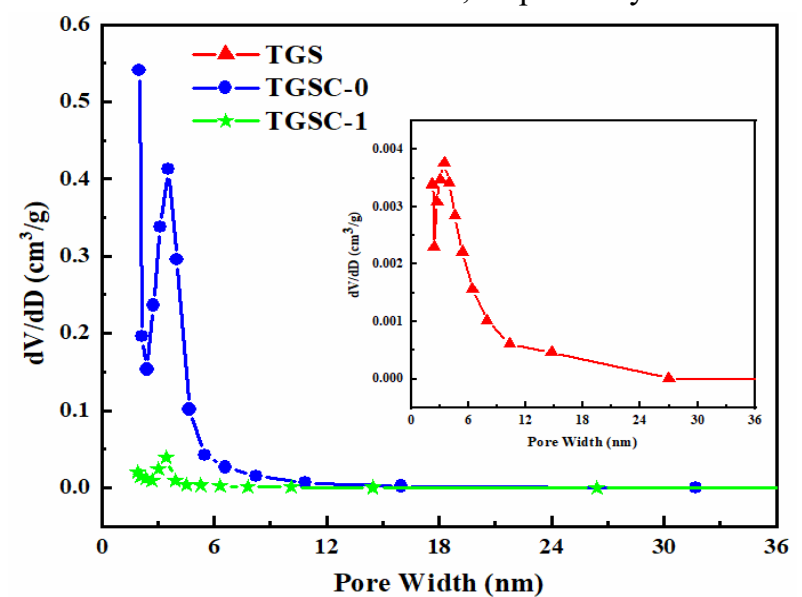

Fig. 6. Pore size distribution

Table 1. Specific surface areas and pore structures of loofah carbon materials.

\begin{tabular}{cccc}
\hline Material & $S_{B E T}\left(\mathrm{~m}^{2} / \mathrm{g}\right)$ & $V_{\text {total }}\left(\mathrm{cm}^{3} / \mathrm{g}\right)$ & $D_{p}(\mathrm{~nm})$ \\
\hline TGS & 5.304 & 0.032 & 9.267 \\
TGSC-0 & 650.904 & 0.727 & 3.963 \\
TGSC-1 & 427.967 & 0.230 & 2.347 \\
\hline
\end{tabular}

\subsection{Adsorption studies}

\subsubsection{Comparison of SCF adsorption by different adsorbents}


The adsorption properties of the three loofah adsorbents on SCF pollutants (oil, particulate matter, and NMHC) were evaluated. Adsorption breakthrough measurements are widely used direct measurements to evaluate adsorption performance (Zeng. et all. 2020). The adsorption breakthrough curves and adsorption capacities of TGS, TGSC-0, and TGSC-1 for SCF pollutants under dry gas-flue are shown in Figures 7 and 8, respectively. Adsorption performance data are also shown in Table 2. Oils, particulate matter (PM2.5 and PM10), and NMHC exhibited the fastest breakthroughs on TGS, with adsorption capacities of $0.767 \mathrm{mg} / \mathrm{g}, 0.792 \mu \mathrm{g} / \mathrm{g}, 1.536 \mu \mathrm{g} / \mathrm{g}$, and $0.533 \mathrm{mg} / \mathrm{g}$, respectively. The adsorption capacities of TGSC-1 were $1.961 \mathrm{mg} / \mathrm{g}, 1.056 \mu \mathrm{g} / \mathrm{g}, 2.111 \mu \mathrm{g} / \mathrm{g}$, and $1.092 \mathrm{mg} / \mathrm{g}$, respectively. The adsorption capacity and saturated adsorption capacity values of TGSC-0 were intermediate to those of TGS and TGSC-1. The adsorption capacities of the three adsorbents thus followed the trend of: TGSC$1>$ TGSC- $0>$ TGS. The primary reason for these differences is that TGSC-0 was prepared by dehydrating TGS at high temperature, leading to the pores being dredged and expanded along with increased specific surface areas, and enhanced adsorption capacity. The pore volumes of TGSC-1 were smaller than those of TGSC-0, while abundant hydroxyl, carbonyl, and carboxyl groups were added to its surface that can effectively chemically adsorb SCF composite pollutants, followed by physical adsorption by TGSC-1. Thus, TGSC-1 exhibited the best adsorption performance.
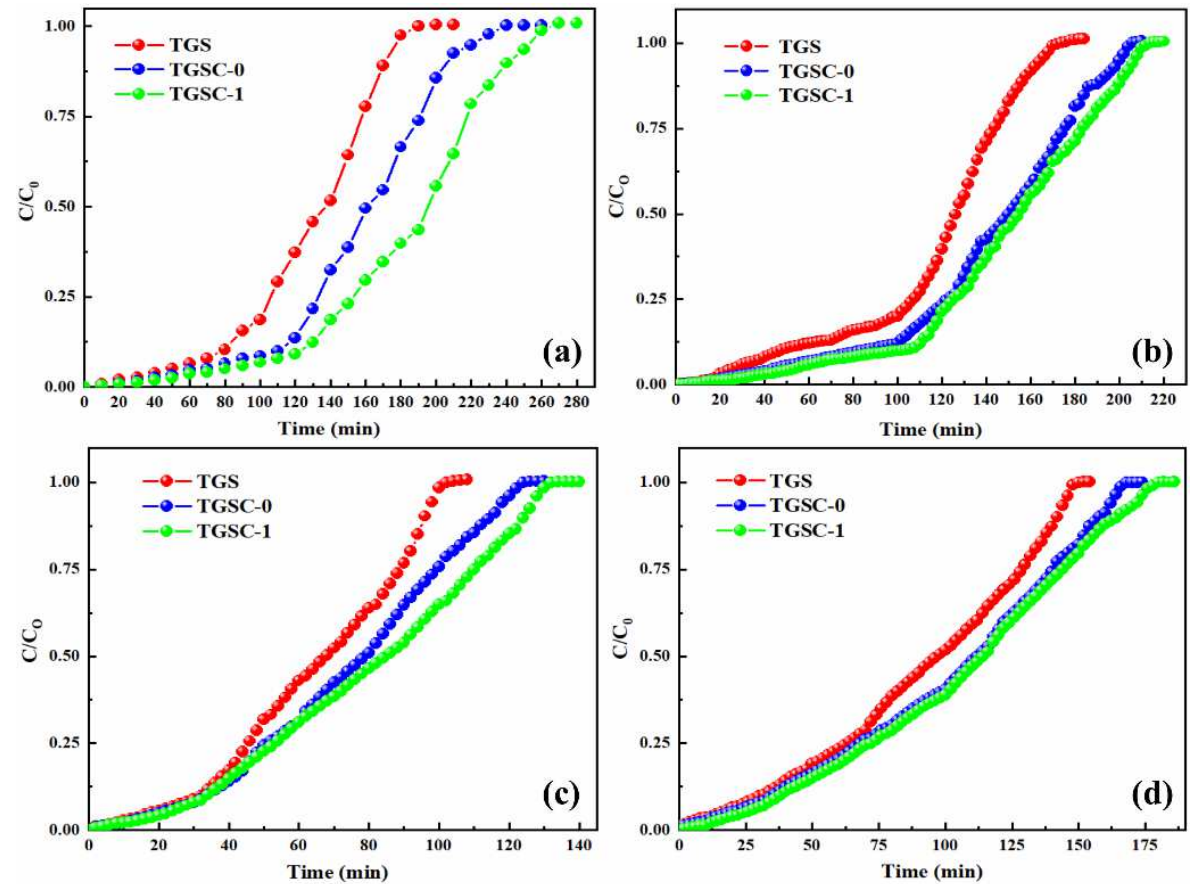

Fig. 7. Adsorption breakthrough curves for different loofah adsorbents and (a) oils, (b) NMHC, (c) PM2.5, and (d) PM10. 

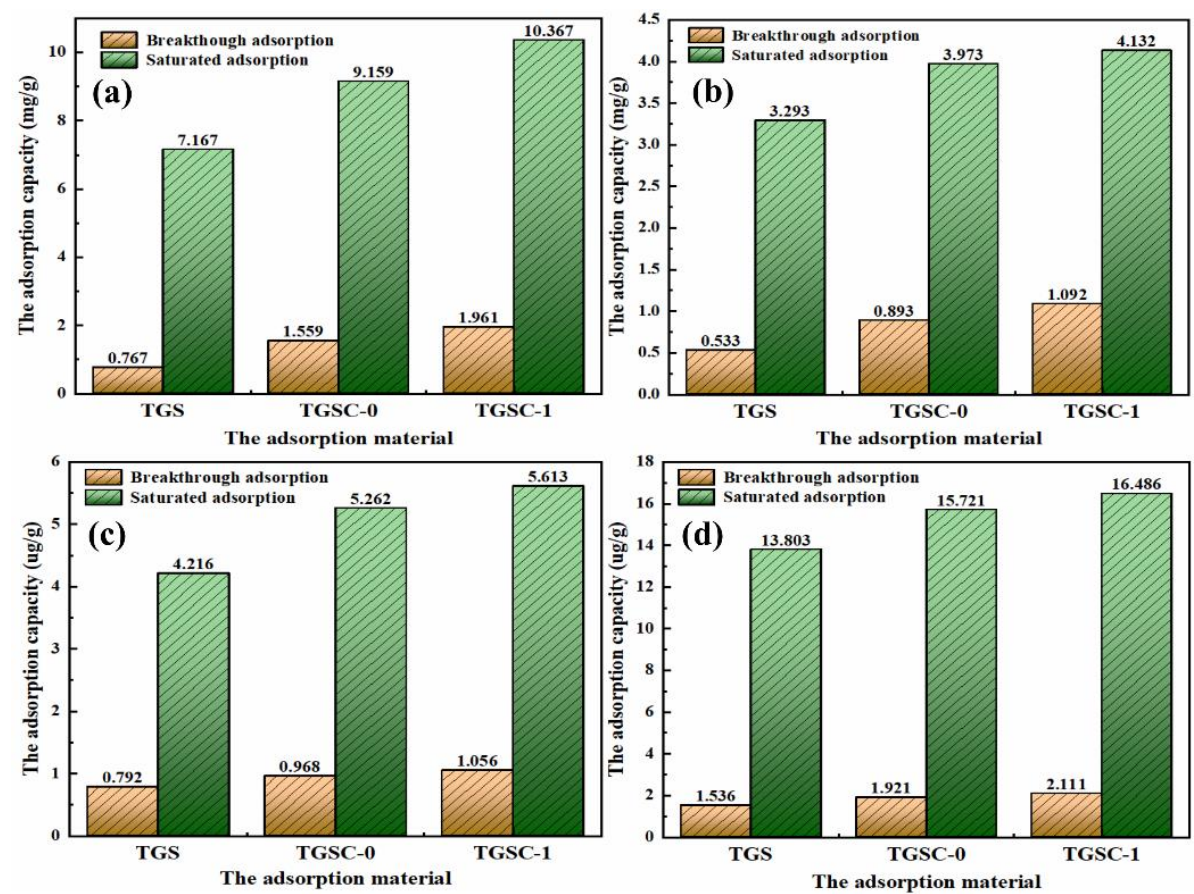

Fig. 8. The adsorption capacity of different loofah adsorbents for (a) oils, (b) NMHC, (c) PM2.5, and (d) PM10.

Table 2. Characteristics of loofah adsorption of SCF pollutants.

\begin{tabular}{cccccc}
\hline Sample & Pollutant & $\begin{array}{c}\text { Breakthrough } \\
\text { time (min) }\end{array}$ & $\begin{array}{c}\text { Breakthrough } \\
\text { adsorption } \\
\text { capacity }\end{array}$ & $\begin{array}{c}\text { Saturated } \\
\text { adsorption } \\
\text { time (min) }\end{array}$ & $\begin{array}{c}\text { Saturated } \\
\text { adsorption } \\
\text { capacity }\end{array}$ \\
\hline \multirow{6}{*}{ TGS } & Oil & 20 & $0.767 \mathrm{mg} / \mathrm{g}$ & 180 & $7.167 \mathrm{mg} / \mathrm{g}$ \\
& NMHC & 28 & $0.533 \mathrm{mg} / \mathrm{g}$ & 166 & $3.293 \mathrm{mg} / \mathrm{g}$ \\
& PM2.5 & 18 & $0.792 \mu \mathrm{g} / \mathrm{g}$ & 96 & $4.216 \mu \mathrm{g} / \mathrm{g}$ \\
& PM10 & 16 & $1.536 \mu \mathrm{g} / \mathrm{g}$ & 144 & $13.803 \mu \mathrm{g} / \mathrm{g}$ \\
\hline \multirow{5}{*}{ TGSC-0 } & Oil & 40 & $1.559 \mathrm{mg} / \mathrm{g}$ & 230 & $9.159 \mathrm{mg} / \mathrm{g}$ \\
& NMHC & 46 & $0.893 \mathrm{mg} / \mathrm{g}$ & 200 & $3.973 \mathrm{mg} / \mathrm{g}$ \\
& PM2.5 & 22 & $0.968 \mu \mathrm{g} / \mathrm{g}$ & 120 & $5.262 \mu \mathrm{g} / \mathrm{g}$ \\
& PM10 & 20 & $1.921 \mu \mathrm{g} / \mathrm{g}$ & 164 & $15.721 \mu \mathrm{g} / \mathrm{g}$ \\
\hline \multirow{5}{*}{ TGSC-1 } & Oil & 50 & $1.961 \mathrm{mg} / \mathrm{g}$ & 260 & $10.367 \mathrm{mg} / \mathrm{g}$ \\
& NMHC & 56 & $1.096 \mathrm{mg} / \mathrm{g}$ & 208 & $4.132 \mathrm{mg} / \mathrm{g}$ \\
& PM2.5 & 24 & $1.056 \mu \mathrm{g} / \mathrm{g}$ & 128 & $5.613 \mu \mathrm{g} / \mathrm{g}$ \\
& PM10 & 22 & $2.111 \mu \mathrm{g} / \mathrm{g}$ & 172 & $16.486 \mu \mathrm{g} / \mathrm{g}$ \\
\hline
\end{tabular}

\subsubsection{Effect of different adsorbent masses on SCF adsorption performance}

The effects of different masses of TGSC-1 on SCF adsorption breakthrough curves are shown in Fig. 9. At TGSC-1 masses of 3, 5, 7, 9, and 11 g, TGSC-1 breakthrough times were 20, 50, 60, 70, and $80 \mathrm{~min}$, respectively. In addition, breakthrough times of TGSC-1 for NMHC were 28, 56, 86, 156, and 206, respectively. Lastly, the breakthrough times of TGSC-1 for PM2.5 were 22, 24, 36, 46, and 62, while the breakthrough times of TGSC-1 for PM10 were 20, 22, 32, 42, and 64 min, respectively. Thus, breakthrough times increased with TGSC-1 mass increases. 
The effects of different adsorbent masses on SCF adsorption performance is shown in Figure 10. At TGSC-1 masses of 3, 5, 7, 9, and $11 \mathrm{~g}$, the breakthrough adsorptions of TGSC-1 to oil were $0.762,1.961$, $2.452,3.936$, and $5.267 \mathrm{mg} / \mathrm{g}$, respectively, while saturated adsorptions were 8.762, 10.367, 13.952, 19.936, and $21.933 \mathrm{mg} / \mathrm{g}$, respectively. Breakthrough adsorptions of TGSC-1 for NMHC were 0.533 , $1.092,2.115,5.153$, and $10.230 \mathrm{mg} / \mathrm{g}$, respectively, while saturated adsorptions were $3.293,4.132,5.765$, 8.220 , and $14.430 \mathrm{mg} / \mathrm{g}$, respectively. The breakthrough adsorptions of TGSC-1 for PM2.5 were 0.790 , $1.056,1.955,3.340$, and 6.770 , respectively, while saturated adsorptions were 4.017, 5.613, 7.345, 10.820, and $19.090 \mu \mathrm{g} / \mathrm{g}$, respectively. The breakthrough adsorptions of TGSC-1 for PM10 were 1.577, 2.111, $3.805,6.673$, and $15.290 \mu \mathrm{g} / \mathrm{g}$, respectively, while saturated adsorptions were 11.977, 16.486, 23.005, 37.073, and $65.690 \mu \mathrm{g} / \mathrm{g}$, respectively. Thus, increased TGSC-1 mass was associated with increased adsorption capacity of TGSC-1 to SCF pollutants.
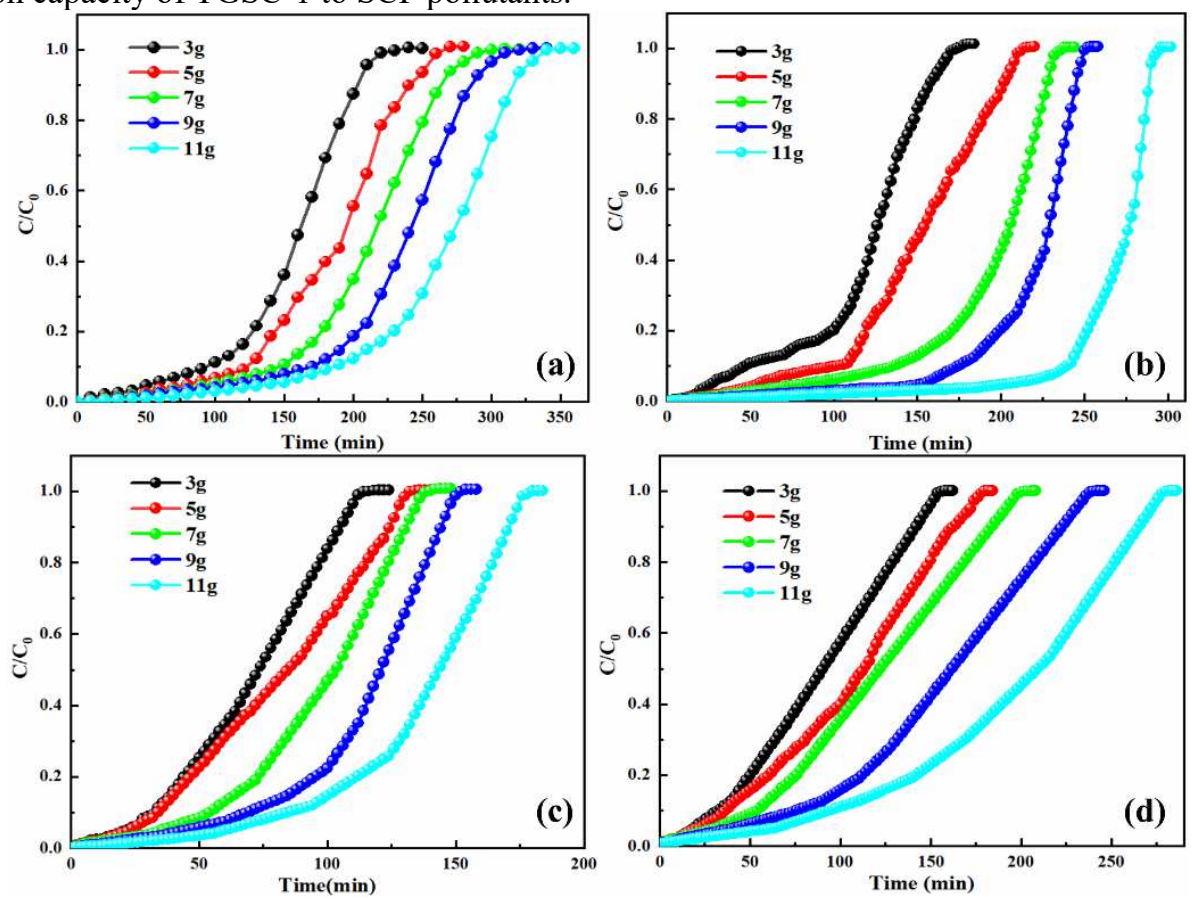

Fig. 9. Effects of different TGSC-1 masses on adsorption breakthrough curves for (a) oils, (b) NMHC, (c) PM2.5, and (d) PM10. 

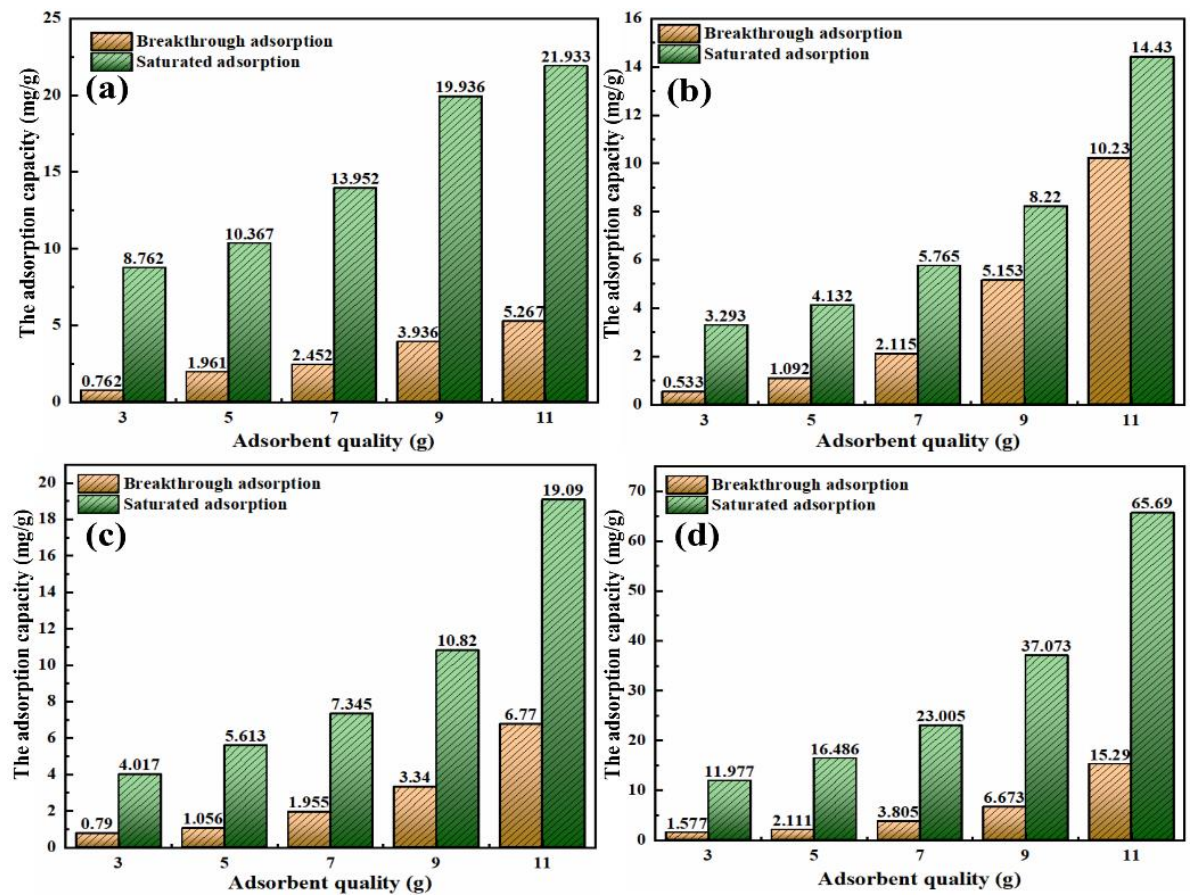

Fig. 10. Effects of different adsorbent masses on adsorption performances for (a) oils, (b) NMHC, (c) PM2.5, and (d) PM10.

The L- $\tau$ fitting line of TGSC-1 adsorption of different pollutants is shown in Fig. 11, which indicates that the breakthrough time for different pollutants varies with the length of TGSC-1 adsorption beds. The parameters of the Wurof equation to evaluate the adsorption of SCF pollutants by TGSC-1 are shown in Table 3.

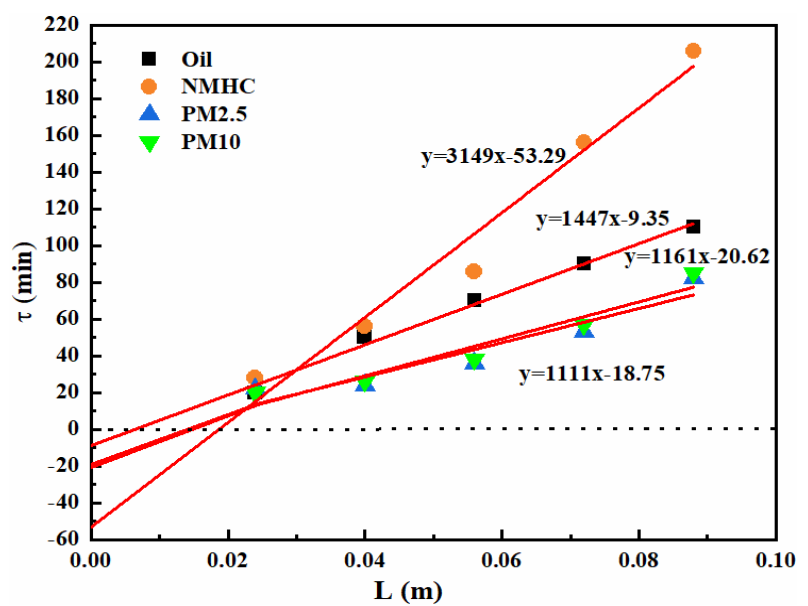

Fig. 11. L- $\tau$ fitting for TGSC-1 adsorption of SCF pollutants.

Table 3. Wurof equation parameters for SCF pollutant adsorption by TGSC-1.

\begin{tabular}{ccccc}
\hline Pollutant & Equation & $K(\mathrm{~min} / \mathrm{m})$ & $\tau_{0}(\mathrm{~min})$ & $R^{2}$ \\
\hline Oils & $\tau=1447 \mathrm{~L}-9.35$ & 1447 & 13.35 & 0.995 \\
NMHC & $\tau=3149 \mathrm{~L}-53.29$ & 3149 & 53.29 & 0.983 \\
PM2.5 & $\tau=1111 \mathrm{~L}-18.75$ & 1111 & 18.75 & 0.987 \\
PM10 & $\tau=1161 \mathrm{~L}-20.62$ & 1161 & 20.62 & 0.992 \\
\hline
\end{tabular}




\subsection{Model fitting}

The Langmuir and Freundlich model fitting curves are shown in Fig. 12 for the adsorption of SCF pollutants (oils, NMHC, PM2.5, and PM10) by three loofah adsorbents (TGS, TGSC-0, and TGSC-1). The $\mathrm{R}^{2}$ values $(0.99116,0.99471$, and 0.99351 , respectively) of the Langmuir model fitting curves were significantly higher than those of the Freundlich model (0.97391, 0.98021, and 0.98953, respectively) for SCF oils. In addition, the $\mathrm{R}^{2}$ values $(0.97814,0.99448$, and 0.99559 , respectively) of the Langmuir model fitting curves were significantly higher than those of the Freundlich model $(0.97174,0.99013$, and 0.98838 , respectively) for NMHC. The $\mathrm{R}^{2}$ values of the Langmuir and Freundlich model fitting curves were all greater than 0.995 , while those of the Langmuir model curve were slightly higher for particulate matter (PM2.5 and PM10). Thus, the Langmuir models better described the adsorption of SCF pollutants by the three loofah adsorbents compared to Freundlich models, indicating that the distribution of adsorption sites on the surfaces of TGS, TGSC-0, and TGSC-1 were generally uniform (Na. et all. 2020).

The Langmuir model is based on the principle of monolayer adsorption that is very suitable for adsorbent surface chemisorption (Alawode. et all. 2019). In contrast, the Freundlich model is more appropriate for non-uniformly distributed adsorption sites on adsorbent surfaces (Na. et all. 2020). The above results suggest that the adsorption of SCF pollutants (oils, NMHC, PM2.5, and PM10) by TGS, TGSC-0, and TGSC-1 was consistent with monolayer adsorption, and that chemical adsorption plays a major role in these processes. 

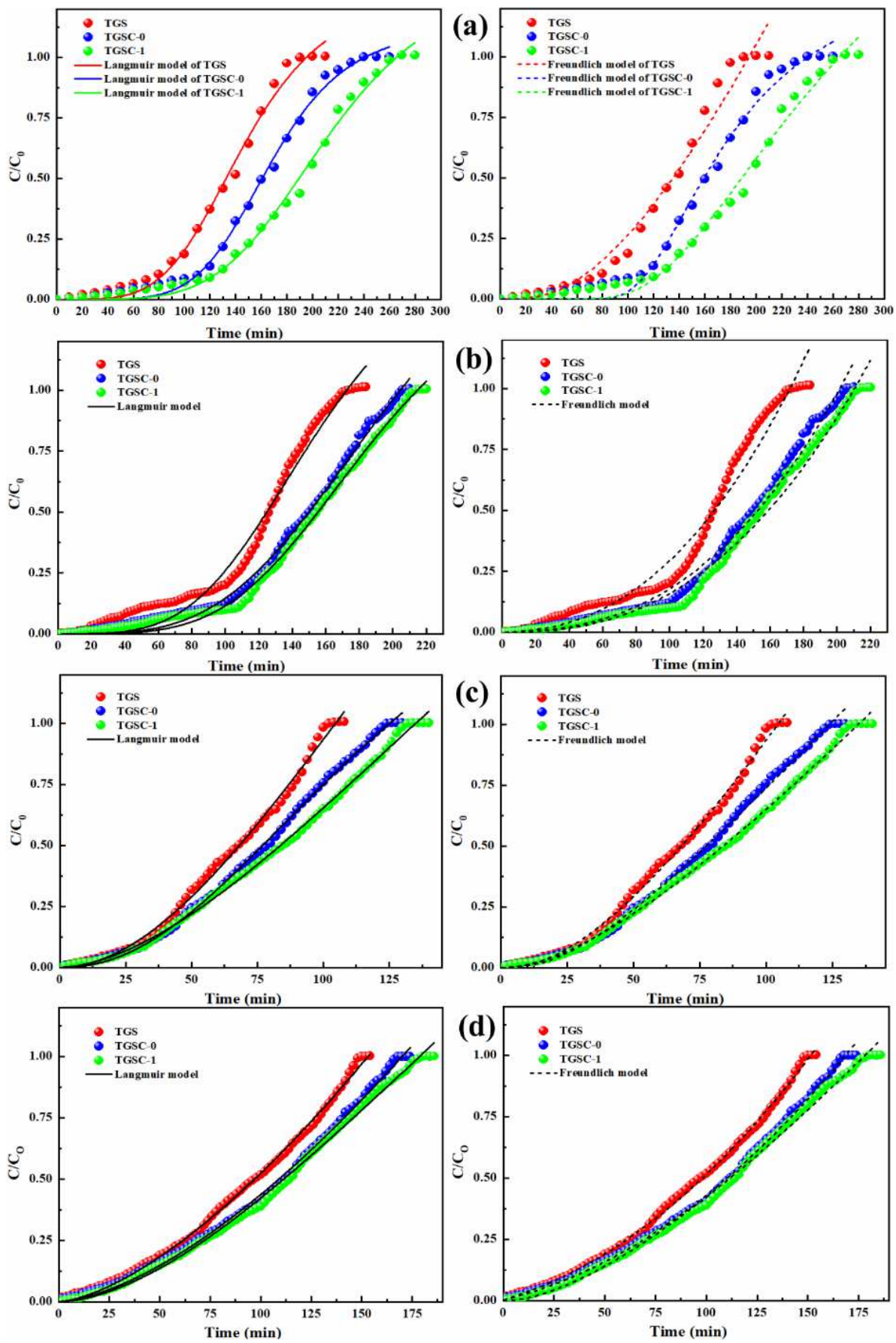

Fig. 12. Langmuir and Freundlich model fitting curves for adsorption of (a) oils, (b) NMHC, (c) PM2.5, and (d) PM10.

\subsection{Adsorption mechanism}

TGS is made up of lignin, semi lignin and other plant fibers, and phosphoric acid can quickly penetrate into TGS after dehydration. So, the full pores were distributed into the TGSC-0, which provides a site for the attachment of functional groups and the adsorption of pollutants (Jawad. et all. 2020). $\mathrm{Fe}^{2+}$ and $\mathrm{Fe}^{3+}$ formed in Fenton reagent have good catalytic effect on the conversion of $\mathrm{H}_{2} \mathrm{O}_{2}$ to $\mathrm{HO}$. and $\mathrm{HOO}$, and $\mathrm{HO} \cdot$ radical has strong addition reaction and oxidation ability because of its lack of electron groups and high potential (Pan. et all. 2008). Therefore, HO and HOO radicals can react with unsaturated double bonds on the surface of TGSC- 0 , resulting in the formation of carbonyl and carboxyl 
functional groups on the surface of TGSC-1, thus realizing the modification of Fenton reagent.

The stretching vibrations of $\mathrm{C}-\mathrm{H}$ of methyl, methylene, and aromatic rings were measured by infrared oil meter at 2,930 $\mathrm{cm}^{-1}, 2,960 \mathrm{~cm}^{-1}, 3,030 \mathrm{~cm}^{-1}$, respectively, in order to obtain the concentration of oil. C-H has the same polarity as the carbonyl and carboxyl groups on the surface of TGSC-1 (Fig. 3), According to the similar phase solubility principle, TGSC-1 was easier to adsorb $\mathrm{C}-\mathrm{H}$, so the adsorption effect of TGSC-1 on oil was better. There was a mesoporous-microporous structure on the surface of TGSC-1, which was dominated by macroporous adsorption when the adsorption pressure increases (Table 1). In addition, TGSC-1 had rough surface, larger specific surface area and larger adsorption area, which can effectively adsorb particulate matter (PM2.5, PM10). In the process of cooking fumes adsorption, the residual $\mathrm{Fe}^{3+}, \mathrm{Fe}^{2+}, \mathrm{H}_{2} \mathrm{O}_{2}$ on the surface of TGSC-1 has strong oxidation (Fig. 4), and these substances can also oxidize and decompose most of NMHC adsorbed on the surface of TGSC-1, thus improving the adsorption performance of TGSC-1.

\section{Conclusions}

In this study, a natural loofah was successfully prepared as an efficient adsorbent (TGSC-1) of SCF pollutants (oils, particulate matter, and NMHC) and was activated by phosphoric acid and modified by Fenton's reagent. The surface structures and properties of TGSC-1 were characterized by scanning electron microscopy, Fourier transform infrared spectroscopy, X-ray powder diffractometry, and $\mathrm{N}_{2}$ adsorption-desorption isotherm modeling. The results demonstrated that TGSC-1 was the best adsorbent compared with TGS and TGSC-0 and exhibited saturated adsorption capacities for oil, NMHC, PM2.5, and PM10 of 10.367, 4.132, 5.613, and $16.486 \mu \mathrm{g} / \mathrm{g}$, respectively. The enhanced adsorption properties of TGSC-1 are due to the large number of hydroxyls, carbonyl, and carboxyl groups in the material, in addition to iron that exists in the amorphous form that is generated on the surface of TGSC-1 from Fenton's reagent reactions. Additional pollutant adsorption experiments were consequently conducted with TGSC-1 and different masses, wherein increased TGSC-1 mass led to increased breakthrough time and adsorption levels. Further, Langmuir models better fit the adsorption dynamics, with most models exhibiting high $\mathrm{R}^{2}$ values for TGSC-1 adsorption of SCF pollutants (i.e., oils, NMHC, PM2.5, and PM10). Thus, the adsorption dynamics were consistent with monolayer adsorption, with chemical adsorption playing a major role. These results provide a theoretical basis for the application of TGSC adsorption technology in the treatment of cooking fumes.

\section{Acknowledgements}

We appreciate the financial support from the National Natural Science Foundation of China (51468011) and the Guangxi 'Bagui Scholar' Construction Project.

\section{Data availability}

All data generated or analyzed during this study are included in this published article.

\section{Author contributions}

Conceptualization, Lei Liao and Shengpeng Mo; Data curation, Hongqiang Wang; Formal analysis, Jianwen Wei; Funding acquisition, Lei Liao and Shengpeng Mo; Investigation, Huan Zhang and Peng Zeng; Methodology, Huan Zhang and Lei Liao; Project administration, Huan Zhang; Resources, Huan Zhang; Software, Peng Zeng and Hongquan Liu; Supervision, Hongqiang Wang; Validation, Huan Zhang and Aimiao Qin; Visualization, Huan Zhang; Writing - original draft, Huan Zhang; Writing - review \& 
editing, Lei Liao and Shengpeng Mo.

\section{Funding}

This study was funded by the National Natural Science Foundation of China (51468011).

\section{Ethics declarations}

Ethics approval and consent to participate

Not applicable.

Consent for publication

Not applicable.

Competing interests

The authors declare no competing interests.

\section{References}

Alves CA., Vicente Estela D., Evtyugina Margarita, Vicente Ana M.P., Sainnokhoi Tsend-Ayush, Kováts Nora. Cooking activities in a domestic kitchen: Chemical and toxicological profiling of emissions[J]. Science of the Total Environment, 2021, 772: 145412.

Ali H, Aniagor Chukwunonso Onyeka, Nasr Mohamed Fathi, Abou-Okeil Ashraf. Efficacy of treated sodium alginate and activated carbon fibre for $\mathrm{Pb}(\mathrm{II})$ adsorption[J]. International Journal of Biological Macromolecules, 2021, 176:201-216.

Ali HJ, Bardhan Mondira,Islam Md. Atikul,Islam Md. Azharul,Syed Hassan Syed Shatir A., Surip S.N., ALOthman Zeid A., Khan Mohammad Rizwan. Insights into the modeling, characterization and adsorption performance of mesoporous activated carbon from corn cob residue via microwave-assisted $\mathrm{H}_{3} \mathrm{PO}_{4}$ activation[J]. Surfaces and Interfaces,2020, 21.

Alawode AJ., Olugbenga A. Falode. Development of a New Type-I Isotherm for Correction of Langmuir Isotherm's Over-estimation of Adsorption at Higher Pressures[J]. Physical Science International Journal, 2019.

Abdullahi KL, Juana Maria Delgado-Saborit, Roy M. Harrison. Emissions and indoor concentrations of particulate matter and its specific chemical components from cooking: A review[J]. Atmospheric Environment, 2013, 71(2):260-294.

Bardestani R, Gregory S. Patience, Serge Kaliaguine. Experimental methods in chemical engineering: specific surface area and pore size distribution measurements-BET, BJH, and DFT[J]. The Canadian Journal of Chemical Engineering, 2019, 97(11).

Cui MJ, Yin-Ping Hu, Bing-Ying Li, Rui-Xue Wang. Effects of non-ionic surfactants on bioethanol production using extracted cellulose from wheat straw and kinetic studies[J]. Biomass Conversion and Biorefinery, 2021(5).

Cheng SY, Gang Wang, Jianlei Lang, Wei Wen, Xiaoqi Wang, Sen Yao. Characterization of volatile organic compounds from different cooking emissions[J]. Atmospheric Environment, 2016, 145(11): 299307.

Chen HP, Guiying Lin, Xianhua Wang, Yingquan Chen, Yingpeng Liu, Haiping Yang, Jingai Shao. Physicochemical properties and hygroscopicity of tobacco stem biochar pyrolyzed at different temperatures[J]. Journal of Renewable and Sustainable Energy, 2016, 8(1):1812.

Dai JD, Atian Xie, Ruilong Zhang, Wenna Ge, Zhongshuai Chang, Sujun Tian, Chunxiang Li, Yongsheng 
Yan. Scalable preparation of hierarchical porous carbon from lignin for highly efficient adsorptive removal of sulfamethazine antibiotic[J]. Journal of Molecular Liquids, 2018, 256.

Fang XY, Esben Strodl, Chuan-An Wu,Li Liu, Xiao-Na Yin, Guo-Min Wen, Deng-Li Sun, Dan-Xia Xian, Hui Jiang, Jin Jing, Yu Jin, Wei-Qing Chen. Maternal cooking during pregnancy may increase hyperactive behaviors among children aged at around 3 years old[J]. Indoor Air, 2020, 30(1).

$\mathrm{Fu}$ YH, Niyu Zhang, Yafei Shen, Xinlei Ge, Mindong Chen. Micro-mesoporous carbons from original and pelletized rice husk via one-step catalytic pyrolysis[J]. Bioresource Technology, 2018, 269:67-73. Fu W, Nie Guanghui, Zhou Bo, Wang Liang, Ma Yifei, Peng Suwan, Ou Songfeng, Qin Jian,Zhang Li'e, Li Shu, Zou Ruosi, Zeng Xiaoyun, Zhang Zhiyong, Zou Yunfeng. Association between Chinese cooking oil fumes and sleep quality among a middle-aged Chinese population[J]. Environmental pollution, 2017, 227(8):543-551.

Guo WW, Li Tianqi, Chen Qianru, Wan Junfeng, Zhang Jie, Wu Bo, Wang Yan. The roles of wavelength in the gaseous toluene removal with center dot $\mathrm{OH}$ from $\mathrm{UV}$ activated Fenton reagent [J]. Chemosphere,2021, 275.

Hoang HP, Lofty Vivian, Basta Altaf, Trens Philippe. Designing microporous activated carbons from biomass for carbon dioxide adsorption at ambient temperature. A comparison between bagasse and rice by-products[J]. Journal of Cleaner Production, 2021, 294.

Ho HA, Shan-Yu Wang, Wei-Hung Chiang, Van-Huy Nguyen, Jun-Long Chiu, Jeffrey C.S. Wu. Moderate-temperature catalytic incineration of cooking oil fumes using hydrophobic honeycomb supported Pt/CNT catalyst[J]. Journal of Hazardous Materials, 2019, 379.

Jawad AH., Bardhan Mondira, Islam Md. Atikul, Islam Md. Azharul, Syed Hassan Syed Shatir A., Surip S.N., ALOthman Zeid A., Khan Mohammad Rizwan. Insights into the modeling, characterization and adsorption performance of mesoporous activated carbon from corn cob residue via microwave-assisted $\mathrm{H}_{3} \mathrm{PO}_{4}$ activation[J]. Surfaces and Interfaces, 2020, 21.

Liang JF, Li Qian Wei, Gao Jun Qin, Feng Jiu Ge, Zhang Xiao Ya, Hao Yi Jing, Yu Fei Hai. Biocharcompost addition benefits Phragmites australis growth and soil property in coastal wetlands[J]. Science of The Total Environment, 2021, 769.

Li XH, Wang Shuxiao, Duan Lei, Hao Jiming, Long Zhengwei. Design of a compact dilution sampler for stationary combustion sources[J]. Journal of the Air \& Waste Management Association (1995), 2011, 61(11):1124-1130.

Vasilyeva M.S., Lukiyanchuk I.V., Yarovaya T.P.,Ustinov A.Yu., Nedozorov P.M.,Fedorets A.N., Arefieva O.D.. Degradation of methyl orange in heterogeneous photo-Fenton reaction over V(IV)containing oxide-phosphate coatings formed on titanium by plasma electrolytic oxidation[J]. Surface \& Coatings Technology, 2021, 410(4):126898.

Ma YS, Deng Linjing, Ma Ping, Wu Yang, Yang Xu, Xiao Fang, Deng Qihong. In vivo respiratory toxicology of cooking oil fumes: Evidence, mechanisms and prevention[J]. Journal of Hazardous Materials, 2020, 402: 123455.

Novi Sri BK, Sumantri Indro. Langmuir and Freundlich isotherm adsorption using activated charcoal from banana peel to reduce total suspended solid (TSS) levels in tofu industry liquid waste[J]. IOP Conference Series: Materials Science and Engineering, 2021, 1053(1):012113 (10pp)

$\mathrm{Na} \mathrm{CZ}$, Jiaying $\mathrm{Xu}$. Freundlich interpretation of $\mathrm{pH}$ control and ion specificity in zeolite cation exchange[J]. SN Applied Sciences, 2020, 2(8):1-7.

Naseri M, Mojtaba Jouzizadeh, Mahsa Tabesh, Milad Malekipirbazari, Raikhangul Gabdrashova, Sholpan Nurzhan, Hamta Farrokhi, Reza Khanbabaie, Hossein Mehri-Dehnavi, Zhibek Bekezhankyzy, 
Aidana Gimnkhan, Maryam Dareini, Akbota Kurmangaliyeva, Nazia Islam,Byron Crape, Giorgio Buonanno, Flemming Cassee, Mehdi Amouei Torkmahalleh. The impact of frying aerosol on human brain activity[J]. Neurotoxicology, 2019, 74:149-161.

Pan, B.; Xing, B. Adsorption Mechanisms of Organic Chemicals on Carbon Nanotubes. Environ. Sci. Technol. 2008, 42, 9005-9013.

Ramalakshmi R., Stella Mary S., Shahil Kirupavathy S.. Influence of metallic dopants on the growth, optical and mechanical properties of pure ammonium oxalate crystals[J]. Optical Materials, 2021, 113.

Song B, Hongxu Liang, Ruru Sun, Pai Peng, Yun Jiang, Diao She. Hydrogel synthesis based on lignin/sodium alginate and application in agriculture $[\mathrm{J}]$. International Journal of Biological Macromolecules, 2020, 144:219-230.

Sinha P, Archit Datar, Chungsik Jeong, Xuepeng Deng, Yongchul G. Chung, Li-Chiang Lin. Surface Area Determination of Porous Materials Using the Brunauer-Emmett-Teller (BET) Method: Limitations and Improvements[J]. The Journal of Physical Chemistry C, 2019, 123(33).

Shen YF, Mindong Chen, Tonghua Sun, Jinping Jia. Catalytic reforming of pyrolysis tar over metallic nickel nanoparticles embedded in pyrochar[J]. Fuel, 2015, 159(11):570-579.

Tucki K, A Bączyk, M Klimkiewicz, J Mączyńska, M Sikora. Comparison of parameters and composition of exhaust fumes of engine fuelled by rapeseed oil and its mixtures with diesel and gasoline[J]. IOP Conference Series: Earth and Environmental Science, 2019, 214(1):012109.

Wang Z, Wang Guangjin, Li Wenyao, Cui Zhe, Wu Jianghong, Akpinar Isil, Yu Li, He Guanjie, Hu Junqing. Loofah activated carbon with hierarchical structures for high-efficiency adsorption of multilevel antibiotic pollutants[J]. Applied Surface Science, 2021, 550:149313.

Wang F, Wang J, Li Y, Han X, Hu H, Yu C, Yuan J, Yao P, Miao X, Wei S, Wang Y, Chen W, Liang Y, Guo H, Zhang X, Yang H, Wu T, He M. Associations between daily cooking duration and the prevalence of diabetes and prediabetes in a middle-aged and elderly Chinese population: A cross-sectional study[J]. Indoor air, 2017, 28(2):238-246.

Wang G, Shui-Yuan Cheng, Wei Wei, Wei Wen, Xiao-Qi Wang, Sen Yao. Chemical Characteristics of Fine Particles Emitted from Different Chinese Cooking Styles[J]. Aerosol and Air Quality Research, $2015,15(6 \mathrm{~S})$.

Wong GWK, Bert Brunekreef, Philippa Ellwood, H Ross Anderson, M Innes Asher, Julian Crane, Christopher KW Lai. Cooking fuels and prevalence of asthma: a global analysis of phase three of the International Study of Asthma and Allergies in Childhood (ISAAC)[J]. The Lancet Respiratory Medicine, 2013, 1(5):386-394.

Yu KP, Ke Ruo Yang, Yen Chi Chen, Jia You Gong, Yen Ping Chen, Hui-Chi Shih, Shih-Chun Candice Lung. Indoor air pollution from gas cooking in five Taiwanese families[J]. Building and Environment, 2015, 93.

Zheng L, Xing Konglong, Rehman Rabia. Removal of Acid Red 88 Using Activated Carbon Produced from Pomelo Peels by $\mathrm{KOH}$ Activation: Orthogonal Experiment, Isotherm, and Kinetic Studies[J]. Journal of Chemistry, 2021, 2021:1-9.

Zheng MM, Yang Yue, Qiao Sen, Zhou Jiti, Quan Xie. A porous carbon-based electro-Fenton hollow fiber membrane with good antifouling property for microalgae harvesting[J]. Journal of Membrane Science, 2021, 626:119189.

Zhang XY, Yonghua He, Quanhui Lin, Lili Huang, Qunwei Zhang, Youqiong Xu. Adverse effects of subchronic exposure to cooking oil fumes on the gonads and the GPR30-mediated signaling pathway in female rats[J]. Molecular \& Cellular Toxicology, 2020, 16(1):13-24. 
Zhao XW, Hua Zhang, Wangming Li, Xingke Li, Wen Fan, Yanyan Zhang. Langmuir-diffusion model: Its modification and further application to glutinous rice flour particles[J]. Journal of Food Process

540 Engineering, 2020(2): e13470.

541 Zeng X, Liu Xi, Li Yu-heng, Rodrigues Hugo. The Breakthrough Time Analyses of Lead Ions in CCL

542 considering Different Adsorption Isotherms[J]. Advances in Civil Engineering, 2020, 2020(2):1-10

543 Zhang DC, Jun-Jie Liu, Li-Zhi Jia, Pan Wang, Xu Han. Speciation of VOCs in the cooking fumes from

544 five edible oils and their corresponding health risk assessments[J]. Atmospheric Environment, 2019,

545 211(8):6-17.

546 Zhao Y, Liu L, Tao P, et al. Review of Effluents and Health Effects of Cooking and the Performance of 547 Kitchen Ventilation[J]. Aerosol and Air Quality Research, 2019, 19(8):1937-1959.

548 Zhang J, Yang Jianpeng, Tian Qing, Liang Xing, Zhu Yanbin, Sand Wolfgang, Li Fang, Ma Chunyan,

549 Liu Yanbiao, Yang Bo. Durability and performance of loofah sponge as carrier for wastewater treatment

550 with high ammonium[J]. Water Environment Research, 2019, 91(7):581-587.

551 Zietzschmann F, Stützer C, Jekel M. Granular activated carbon adsorption of organic micro-pollutants in

552 drinking water and treated wastewater - Aligning breakthrough curves and capacities[J]. Water Research, 553 2016, 92:180-187. 


\section{Supplementary Files}

This is a list of supplementary files associated with this preprint. Click to download.

- GraphicalAbstract.docx 